\title{
Accessory penis: A rare method of peno-urethral separation of sexual function and voiding following successful complex hypospadias reconstruction with a free ileum flap
}

\author{
Rossella Elia ${ }^{1,2}$, Georgios Pafitanis ${ }^{1,3}$, Pedro Ciudad ${ }^{1}$, Hung-Chi Chen ${ }^{1}$ \\ ${ }^{1}$ Department of Plastic and Reconstructive Surgery, China Medical University Hospital, Taichung, Taiwan; ${ }^{2}$ Division of Plastic and \\ Reconstructive Surgery, Department of Emergency and Organ Transplantation, University of Bari, Bari, Italy; ${ }^{3}$ Group for Academic Plastic \\ Surgery, The Royal London Hospital, Barts Health NHS Trust, Queen Mary University of London, London, UK
}

\begin{abstract}
Hypospadias is a congenital malformation of the male genitalia. The reconstructive objectives are to obtain voiding with laminar flow and satisfactory sexual function. Several urethroplasty techniques have been described, but for perineal or revisional cases no single technique has shown robust success. In this study, we describe the expanded use of intestinal flaps for urethral reconstruction and report a peculiar request from a patient to undergo peno-urethral separation after successful hypospadias repair with a free ileum flap. A 51-year-old male patient with perineal hypospadias underwent several urethral reconstructive procedures with poor outcomes. A free ileum flap was attempted as a substitute for the urethra. Following successful reconstruction, separation of the neo-urethra (ileum) from the penile body was performed to address the patient's sexual expectations. A free ileum flap proved to be a reliable urethral substitute in perineal hypospadias reconstruction, with a successful outcome. The peno-urethral separation with the creation of an "accessory penis," however peculiar, optimized the results in terms of both sexual and urinary function. Anatomical restoration of the urethra and patient-reported expectations are the key to successful hypospadias reconstructive procedures. Sexual function outcomes and the patient's perception of success should not be underestimated, even when urinary function has been restored.
\end{abstract}

Keywords Hypospadias / lleum / Urethra / Penis

Received: 11 Jun 2018 • Revised: 1 Mar $2019 \bullet$ Accepted: 23 Apr 2019

pISSN: 2234-6163 • elSSN: 2234-6171 • https://doi.org/10.5999/aps.2018.00689 • Arch Plast Surg 2019;46:381-385
Correspondence: Rossella Elia Division of Plastic and Reconstructive Surgery, University of Bari, Piazza Giulio Cesare, Bari 70124, Italy

Tel: $+39-3409616476$ Fax: +39-0805714030 E-mail: rossellaelia4@gmail.com

\section{INTRODUCTION}

Hypospadias is a common congenital malformation, although its true prevalence is difficult to estimate, and the surgical repair to re-establish anatomical micturition may have long-term so- cio-sexual impacts in males [1]. Hypospadias occurs when the development of the urethral spongiosum and prepuce halts, resulting in a meatal location proximal to that of the distal glans. The more severe the hypospadias, the more it is accompanied by chordee and penoscrotal transposition. 
More than 300 different surgical repairs of hypospadias have been reported [2]. The goal of hypospadias repair is to restore the slit-like terminal meatus to allow standing micturition and satisfactory sexual function. Baskin and Ebbers [3] delineated five surgical steps common to all hypospadias repair techniques: (1) orthoplasty, (2) urethroplasty, (3) meatoplasty and glanuloplasty, (4) scrotoplasty, and (5) epithelial coverage. Contemporary hypospadias repair is very successful, but severe cases may require multiple revisions due to complications such as ectopic meatus, urethral fistula, urethral stricture, and ventral penile curvature $[4,5]$. In more complex procedures or revisional reconstructions, conventional techniques fail to restore urethral continuity, and a free intestinal flap may provide a salvage solution [6-8].

Complex hypospadias can affect both the physical and psychosexual development of an individual [9]. Herein, we report our experience of complex hypospadias repair with a free ileum flap, detailing the technical aspects of intestinal urethral reconstruction and considerations of both urination and sexual function, along with a detailed description of the first case of a peculiar "accessory penis" formation designed to address the patient's satisfaction with the outcomes involving sexual function.

\section{CASE}

A 51-year-old man with perineal congenital hypospadias underwent Bracka orthourethroplasty and staged Nesbit plication with correction of the penile shaft chordae. He subsequently underwent several failed urethroplasty procedures. He reported straining during micturition due to formation of a urethral stricture. He presented at Department of Plastic and Reconstructive Surgery of China Medical University Hospital, complaining of recurrent episodes of urinary retention, urethral stricture, and a ventral scar with a lack of preputial or redundant penile skin.

Urethral augmentation using local options was contraindicated and substitution urethroplasty was required. The first free ileum flap urethroplasty, a ventral skin resurfacing using a local rotational flap to cover the microvascular anastomoses, and a split-thickness skin graft (STSG) were performed to reconstruct the urethra, penis, and scrotum. This achieved successful shortterm voiding. One year later, the patient was re-admitted for a residual fistula between the ileum and the native urethra. The urethra-cutaneous fistula was excised and the ileum-urethra anastomosis was reinforced by a pedicled left gracilis muscle flap. Normal micturition in the standing position and adequate sexual function were reported during 3 years of follow-up. However, ventral skin scar contracture prevented the penile shaft from achieving a full-length erection at the STSG-rotational flap junction, and the ileum prolapsed through the penile tip. Scar contracture release, gracilis muscle debulking, and a local rotational flap from the left thigh increased the amount of longitudinal penile ventral skin. The distal part of the ileum was everted to the position of the glans.

Successful normal voiding and the patient's reported satisfaction with both the aesthetic appearance and functional outcomes were maintained for 1 year. Nevertheless, he was concerned and persistently requested a peno-urethral separation. Following a psychological assessment to address the rationale of a further surgical separation procedure, the "accessory penis" method was applied to satisfy the patient's request. Written informed consent was obtained from the patient prior to surgery.

\section{Free ileum flap for urethral substitution in perineal hypospadias repair}

The key steps of using a free ileum flap to reconstruct a case of scarred, stenotic urethral hypospadias when conventional techniques are contraindicated involve resection of the urethra distal to the stricture, flap harvesting, microvascular transfer, and ileum neo-urethra inset. A midline central mini-laparotomy with adequate exposure to the lower abdomen was obtained. The terminal ileum was identified by locating the ileocecal valve (the junction between the ileum and colon). A segment of ileum was isolated and mobilized along a mesenteric blood supply that was sufficiently long to enable a pedicle with length and vessel caliber matching that of the recipient. The segment of ileum was isolated on the selected pedicle via ligation with hemoclips or ligation sutures through the arcade and vasa recta vessels. The ileum was externalized and transected, and bowel continuity was restored via end-to-end two-layer anastomosis using 3/0 polydioxanone (PDS) sutures. The free ileum flap was transferred to the urethral defect and anastomosed to the proximal perineal urethral stump through the laid-open penile shaft with 4/0 PDS sutures. A urinary catheter was inserted across the junction between the ileum and native urethra as a stent and for drainage of urine. PDS was chosen due to the strength of the suture material, to ensure that the suture would not be disrupted when the stent catheter was pulled out. The deep inferior epigastric artery and vein recipient vessels were dissected superiorly and transposed inferiorly to reach the mesenteric free ileum vessels. Microvascular end-to-end anastomoses using $8 / 0$ or $9 / 0$ nylon sutures were performed under microscopic magnification $(\times 10-15)$. Additional steps included closure of the midline laparotomy with $1 / 0$ loop nylon sutures, closure of the laidopen penile shaft, and inset of the distal ileum to the distal glans-neo-urethral external meatus (Fig. 1). 


\section{Fig. 1. Free ileum flap urethroplasty}

(A) Preoperative view of the patient affected by scared stenotic urethral hypospadias. (B) First surgical step: resection of the distal to the stricture urethra. (C) Harvest of the free ileum flap (10 cm length). (D) lleum-neo-urethra inset after microvascular anastomosis end-toend of mesenteric vessels to the deep inferior epigastric artery and vein.
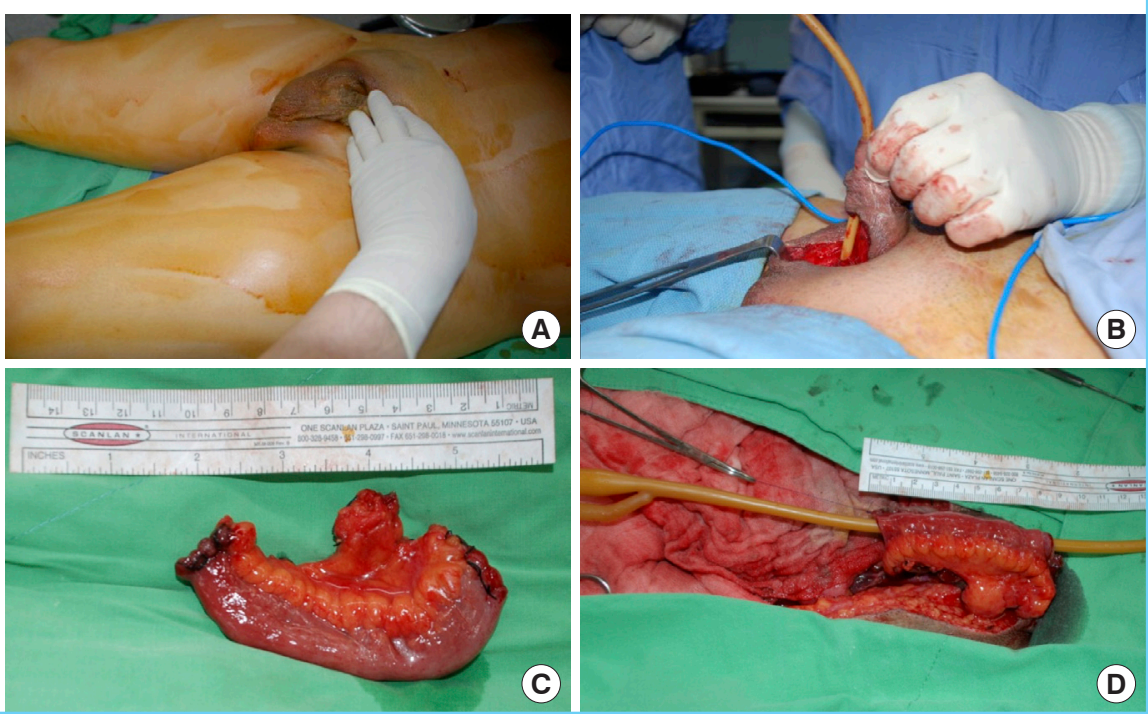

\section{Fig. 2. Before and after peno-urethral separation}

(A) Schematic drawing of the patient's condition after using a segment of ileum as a free flap for reconstruction of the penile urethra. (B) Schematic drawing of the patient's condition after penourethral separation. The patient felt that penile erection was limited, which could have been related to the mesentery of the ileum. After the separation, the patient reported recovery of penile erection with good sensation of the glans and penile skin. The passage of urine was smooth without narrowing or infectious episodes.
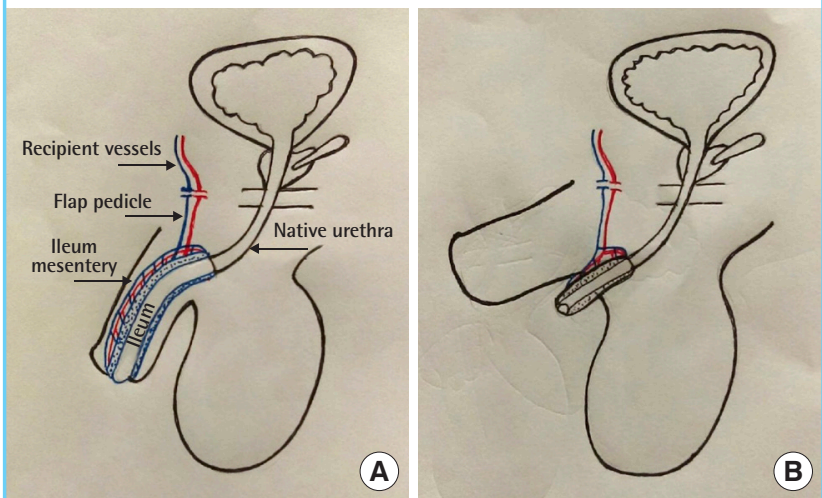

\section{The "accessory penis" method}

A further surgical separation procedure-the "accessory penis" method-was performed to satisfy the patient's request. This step involved the division of the distal neo-urethra (distal ileum) from the penile shaft and ventral-circumferential enterolysis dissection to enable separation from the penile corpus cavernosum. During enterolysis, a precise technique is required to prevent damage to the mesenteric vascular supply. Separation and re-location of the neo-urethral ileum, along with ectopic urethrostomy, were performed inferiorly along the perineal midline. A local fasciocutaneous rotation flap was used to recruit

\section{Fig. 3. Patient's genitalia before peno-urethral separation}

Preoperative view. The picture shows the patient's external genitalia after multiple procedures for hypospadias repair and reconstruction of a functional urethra with a free ileum segment.

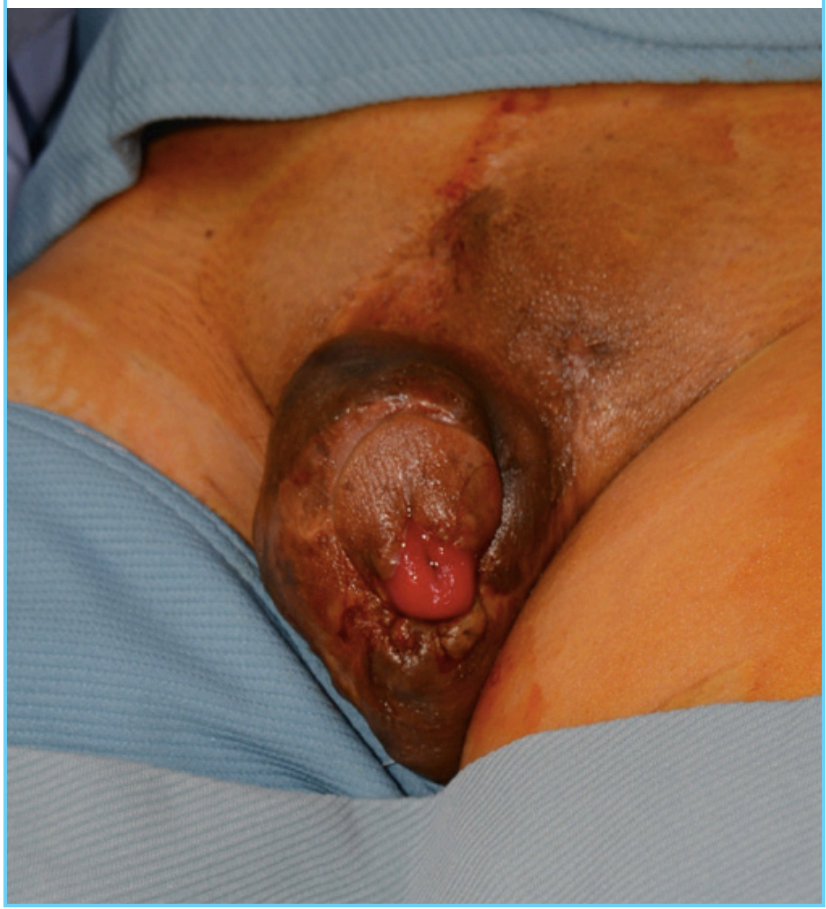

skin length to the penile shaft ventrally, and an STSG provided skin coverage at the base of the penis and the scrotum. The separated ileum segment in the ectopic neo-urethrostomy position had the appearance of an "accessory penis" (Figs. 2-4).

\section{Follow-up and outcomes}

The ectopic neo-urethra (ileum) enabled normal micturition in 


\section{Fig. 4. Peno-urethral separation}

Postoperative view. Separation of the neo-urethra from the penis shaft. Elongation of the penis with a local fasciocutaneous flap and coverage of the neo-urethra with a split-thickness skin graft.

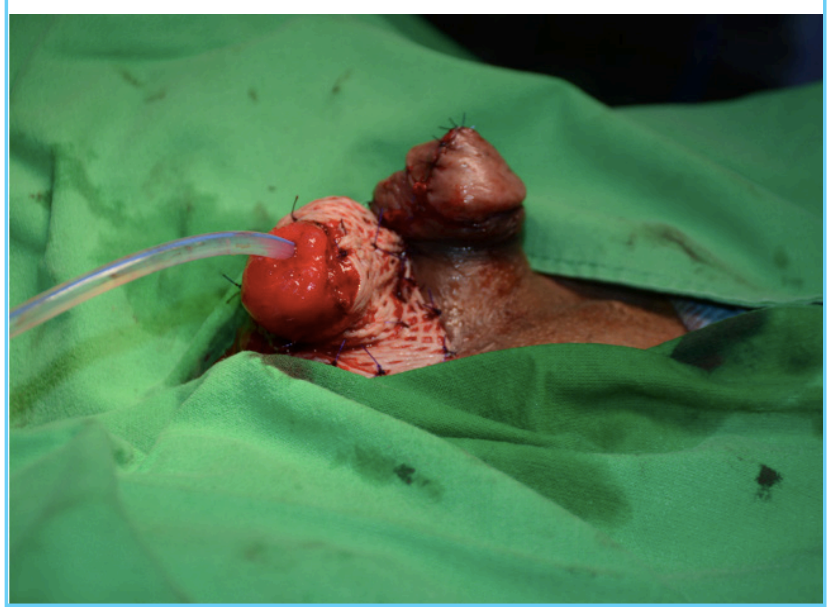

the standing position without post-void dribbling. At a 6-month follow-up, the patient was satisfied with the sexual function of his penis, and reported good erectile function and overall voiding outcomes.

\section{DISCUSSION}

The aim of hypospadias reconstruction is to restore normal penile voiding and sexual function. Several techniques have been described to correct hypospadias $[2,10]$. Tubularized incisional plate urethroplasty has gained widespread acceptance worldwide [10], but for proximal hypospadias or revisional procedures, no single surgical technique provides reliable outcomes due to the complexity these procedures entail. Local, skin, or mucosal grafts are often required to provide additional tissue for restoring urethral continuity, for ventral lengthening, or for skin coverage.

The reconstructive armamentarium of both genitourinary and reconstructive microsurgeons has become broader in recent years, as a deeper understanding of penile blood flow and the application of tissue transfer techniques have improved the overall success of managing complicated cases $[6-8,11]$. Urethral substitution procedures with a free intestinal flap have previously been attempted as a reconstructive alternative when other conventional options failed to provide adequate outcomes. The use of intestinal substitutes for urothelium has been previously reported in the form of ileal conduits and continent reservoirs [12]; however, a literature review yielded few cases where a free intestinal flap was used for urethral reconstruction [6-8]. Bales et al. [6] reported two cases of urethral substitution using a jejunal free flap, while Hiradfar et al. [7] reported a modified substitution urethroplasty using a free appendix flap. To our knowledge, we describe the first reported case of a free ileum flap for urethral substitution following multiple complex, failed hypospadias reconstructions. An intestinal flap should be considered when no other urethral reconstruction method can be safely implemented. The major advantage of using a segment of ileum for urethra reconstruction instead of a segment of jejunum or appendix is that it provides a perfect match in terms of diameter, characteristics of the mucosa, and the option to tailor the intestinal segment to match normal anatomical dimensions. The use of a distal monitoring segment allows safe monitoring of the buried free flap during perioperative care.

An optimal outcome in terms of restoring urinary function in complex hypospadias reconstruction can be considered the key for overall surgical success. Indeed, Meeks et al. [13] published a systematic review revealing that $75 \%$ of the academic literature on urethral reconstruction between 2000 and 2008 defined surgical success as the absence of a secondary procedure, regardless of urinary symptoms. The main advantage of this approach is that it requires very little time or effort on either the patient's or the provider's part. Cystoscopy and retrograde urethrography are objective ways to monitor a reconstructed urethra. However, these methods yield very little insight into a patient's satisfaction with the final reconstruction. Moreover, the literature on the outcomes of pediatric hypospadias repair has established excellent short-term results, but the majority of hypospadias studies lack follow-up into adulthood and do not discuss the psychosexual aspect of hypospadias repair. For children and adolescents, the Child Behavior Checklist has been used, while in adults, the Minnesota Multiphasic Perception Inventory is often used to evaluate psychological questions [14]. The Pediatric Penile Perception Score (PPPS) is an instrument developed for the assessment and comparison of penile appearance. It was validated in boys aged 6 to 17 years for use with hypospadias patients. This validated instrument has also been used in adults. Rynja et al. [15] administered the PPPS to 116 hypospadias patients and 151 control participants, and found that the hypospadias patients had significantly lower satisfaction with penile length than the controls.

The experience reported herein contributes to the discussion about the creation of a standard regimen for the psychological follow-up of men with hypospadias and their partners, and the need to validate a questionnaire to assess the outcomes of the procedures related to sexual function. Peno-urethral separation with the creation of an "accessory penis," as reported in our case, was performed to satisfy several requests by the patient during 2 years of follow-up at 6-month intervals. However unusual, only 
the final surgical reconstruction fully satisfied the patient with penile appearance and erectile function.

In conclusion, anatomical considerations and patient-reported outcomes are both key factors determining the success of hypospadias reconstruction. This study reports the first free ileum flap used as a urethral substitute in a case of extensive urethral loss following congenital hypospadias, after conventional techniques failed to restore function, suggesting that intestinal flaps may prove to be a reliable option for functional long-term outcomes. Peno-urethral separation, however peculiar, was used by the authors as a method to address the patient's reported sexual expectations. The outcomes of hypospadias reconstruction necessitate lifelong follow-up, and success is not only measured in terms of voiding function, but also in terms of cosmetic appearance and sexual function in accordance with the patient's expectations.

\section{NOTES}

\section{Conflict of interest}

No potential conflict of interest relevant to this article was reported.

\section{Ethical approval}

The study was performed in accordance with the principles of the Declaration of Helsinki. Written informed consent was obtained.

\section{Patient consent}

The patient provided written informed consent for the publication and the use of his images.

\section{Author contribution}

Conceptualization: Elia R. Data curation: Elia R, Pafitanis G. Formal analysis: Elia R, Pafitanis G. Methodology: Elia R, Pafitanis G. Project administration: Elia R, Pafitanis G, Chen HC. Visualization: Ciudad P. Writing - originall draft: Elia R. Writing review \& editing: Pafitanis G, Ciudad P, Chen HC. Approval of the final manuscript: all authors.

\section{ORCID}

Rossella Elia https://orcid.org/0000-0002-6094-5159 Georgios Pafitanis https://orcid.org/0000-0003-4712-277X

\section{REFERENCES}

1. Springer A, van den Heijkant M, Baumann S. Worldwide prevalence of hypospadias. J Pediatr Urol 2016;12:152.

2. Snodgrass W, Bush N. Primary hypospadias repair techniques: a review of the evidence. Urol Ann 2016;8:403-8.

3. Baskin LS, Ebbers MB. Hypospadias: anatomy, etiology, and technique. J Pediatr Surg 2006;41:463-72.

4. Barbagli G, De Angelis M, Palminteri E, et al. Failed hypospadias repair presenting in adults. Eur Urol 2006;49:88794.

5. Aldamanhori R, Chapple CR. Management of the patient with failed hypospadias surgery presenting in adulthood. F1000Res 2017;6:1890.

6. Bales GT, Kuznetsov DD, Kim HL, et al. Urethral substitution using an intestinal free flap: a novel approach. J Urol 2002;168:182-4.

7. Hiradfar M, Shojaeian R, Sharifabad PS. Two staged modified substitution urethroplasty using appendix-free flap. BMJ Case Rep 2015;2015:bcr2015210771.

8. Kumar J, Sen S, Dange A, et al. Use of ileum in reconstruction of the congenitally abnormal posterior urethra in two children. J Pediatr Urol 2007;3:333-6.

9. Mondaini N, Ponchietti R, Bonafe M, et al. Hypospadias: incidence and effects on psychosexual development as evaluated with the Minnesota Multiphasic Personality Inventory test in a sample of 11,649 young Italian men. Urol Int 2002; 68:81-5.

10. Wallis MC, Braga L, Khoury A. The role of flaps and grafts in modern hypospadiology. Indian J Urol 2008;24:200-5.

11. Chen SH, Yeong EK, Tang YB, et al. Free and pedicled appendix transfer for various reconstructive procedures. Ann Plast Surg 2012;69:602-6.

12. Neal DE. Replacement of the urinary bladder. Lancet 1999; 353 Suppl 1:SI24-5.

13. Meeks JJ, Erickson BA, Granieri MA, et al. Stricture recurrence after urethroplasty: a systematic review. J Urol 2009; 182:1266-70.

14. Deibert CM, Hensle TW. The psychosexual aspects of hypospadias repair: a review. Arab J Urol 2011;9:279-82.

15. Rynja SP, Wouters GA, Van Schaijk M, et al. Long-term followup of hypospadias: functional and cosmetic results. J Urol 2009;182(4 Suppl):1736-43. 